Research Paper

\title{
KLF6 inhibited oral cancer migration and invasion via downregulation of mesenchymal markers and inhibition of MMP-9 activities
}

Li-Sung Hsu', 2*, Ren-Hung Huang ${ }^{3 *}$, Hung-Wen Lai ${ }^{4,5}$, Hui-Ting Hsu ${ }^{3,6}$, 7, 8, Wen-Wei Sung 6, 7, 8, 9, Ming-Ju

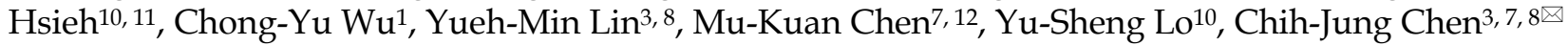

1. Institute of Biochemistry, Microbiology, and Immunology, Chung Shan Medical University, Taichung, Taiwan;

2. Clinical Laboratory, Chung Shan Medical University Hospital Taichung, Taiwan

3. Department of Surgical Pathology, Changhua Christian Hospital, Changhua, Taiwan;

4. Department of Surgery, Changhua Christian Hospital, Changhua, Taiwan;

5. School of Medicine, National Yang Ming University, Taipei, Taiwan;

6. Institute of Medicine, Chung Shan Medical University, Taichung, Taiwan;

7. School of Medicine, Chung Shan Medical University, Taichuang, Taiwan;

8. Department of Medical Technology, Jen-Teh Junior College of Medicine, Nursing and Management, Miaoli, Taiwan;

9. Department of Medical Education, Chung Shan Medical University Hospital, Taichung, Taiwan;

10. Cancer Research Center, Changhua Christian Hospital, Changhua, Taiwan;

11. Graduate Institute of Biomedical Sciences, China Medical University, Taichung, Taiwan;

12. Department of Otorhinolaryngology, Head and Neck Surgery, Changhua Christian Hospital, Changhua, Taiwan.

* These authors contributed equally.

$\triangle$ Corresponding author: Dr. Chih-Jung Chen M.D., Ph.D. Department of Surgical Pathology, Changhua Christian Hospital, 135, Nan-Hsiao Street, Changhua, Taiwan email: 132540@cch.org.tw, Tel: +886-4-7238595 ext 4832

(C) Ivyspring International Publisher. This is an open access article distributed under the terms of the Creative Commons Attribution (CC BY-NC) license (https://creativecommons.org/licenses/by-nc/4.0/). See http://ivyspring.com/terms for full terms and conditions.

Received: 2017.01.03; Accepted: 2017.03.15; Published: 2017.04.09

\begin{abstract}
Krüppel-like factors can bind to specific DNA motifs and regulate various cellular functions, such as metabolism, cell proliferation, and differentiation. Krüppel-like factor 6 (KLF6), a member of this family, is downregulated in human cancers. Oral cancer is a highly prevalent type in Taiwan. Although KLF6 overexpression in human cancer cells inhibits cell proliferation, induces apoptosis, and attenuates cell migration, the effects of KLF6 on oral cancer remains poorly elucidated. This study investigated the role of KLF6 in oral cancer tumorigenesis. Immunohistochemical staining revealed that nuclear KLF6 level was significantly and inversely associated with tumor size and stages. KLF6 overexpression attenuated the migration and invasion of oral cancer SAS cells. Zymography assay demonstrated that KLF6 inhibited the activities of matrix metalloproteinase 9 (MMP-9) and weakened the expression of mesenchymal markers, such as snail, slug, and vimentin. Our study is the first to provide demonstrate that KLF6 functions as a tumor suppressor gene and prevents the metastasis of oral cancer cells.
\end{abstract}

Key words: kruppel-like factor 6 (KLF6), oral cancer, migration, matrix metalloproteinase 9.

\section{Introduction}

Krüppel-like factors (KLFs) are highly conserved zinc-finger proteins that regulate cellular transcription machinery $[1,2]$. KLFs regulate a wide range of cellular functions, including cell proliferation, apoptosis, differentiation, and neoplastic transformation, by binding to GC-rich promoter regions $[1,2]$.
KLF6 functions as a tumor suppressor gene and increases p21 expression via p53-independent pathway [3]. The loss of KLF6 expression has been observed in several human cancers [4-7]. Epigenetic KLF6 alternation in hepatocellular carcinomas has also been detected [8]. KLF6 is downregulated in 85\% of primary non-small cell lung cancers; however, 
forced KLF6 expression in lung cancer cell lines can trigger cells to undergo apoptosis and reduce colony formation ability [6]. The loss of KLF6 expression is also correlated with cancer progression, tumor recurrence, and short survival time in head and neck carcinomas [7]. Exposure to diethyl nitrosamine can induce more hepatic tumors in KLF6-/+ mouse than in wild-type animals [9]. KLF6 downregulation enhances MDM2 gene expression that deregulates the p53 pathway [9]. In prostate cancer, wild-type KLF6 is downregulated through promoter hypermethylation in cancerous parts compared with normal parts [5]. KLF6 overexpression also triggers apoptosis and inhibits osteosarcoma cell migration, whereas KLF6 knockdown reverses these phenomena [10].

Oral squamous cell carcinoma is a common fatal malignancy in Taiwan and the leading cause of cancer-related death worldwide; this malignancy is characterized by specific etiologies, including tobacco product use, alcohol consumption, and human papillomavirus infection [11]. Decreased nuclear KLF4 expression is correlated with poor prognosis and high proliferative activity in oral cancer patients [12]. Although the role of KLF6 in head and neck cancer has been investigated [7], its function in oral cancer patients has yet to be elucidated. In this study, KLF6 was observed to function as an anti-metastasis protein in oral cancer by inhibiting migration and invasion through the downregulation of matrix metalloproteinase-9 (MMP-9). KLF6 also reversed epithelial-to-mesenchymal transition (EMT).

\section{Materials and Methods}

\section{Materials}

All chemicals were purchased from Sigma Aldrich ((St. Louis, MO, USA). Anti-KLF6 and $\beta$-actin was obtained from Santa Cruz (Santa Cruz Biotechnology Inc., Santa Cruz, CA, USA). Anti-E-cadherin, snail, twist, and slug were obtained from GeneTeX (Taipei, Taiwan). RPMI, fetal bovine serum (FBS), penicillin, and streptomycin were purchased from Invitrogen (Thermo Fisher Scientific, Inc., Waltham, MA, USA).

\section{Immunohistochemical stain of KLF6 in oral cancer samples}

A total of 297 oral cancer samples were collected from patients who underwent surgical resection at the Department of Surgery, Changhua Christian Hospital. Tissue microarrays were constructed from paraffin blocks. The stages and grades were classified according to the TNM and World Health Organization classification systems. Immunohistochemical stain was performed using
anti-KLF6 antibody. The intensity of nuclear staining of KLF6 protein was scored semi-quantitatively using scores according to the previously described. The staining intensity of the staining was scored ranging from 0 to 4 . The intensity was classified as either weak $(<2)$ or strong $(\geqq 2)$. The histopathological and clinical data were obtained from the cancer registry of Changhua Christian Hospital. Disease-free survival was measured as the time interval between the surgical operation and either the date of death or the end of follow up. This research was approved by the internal review board of Changhua Christian Hospital.

\section{Cell culture}

Human oral cancer SAS cells were cultured in DMEM/F12 supplemented with $10 \%$ fetal bovine serum, $100 \mu \mathrm{g} / \mathrm{ml}$ of streptomycin and $100 \mathrm{U} / \mathrm{ml}$ of penicillin. The cells were kept at $37^{\circ} \mathrm{C}$ in a humidified incubator with $5 \% \mathrm{CO} 2$.

\section{Western blot analysis}

Total Protein $(50 \mu \mathrm{g})$ derived from SAS cells transfected with pEGFP alone or pEGFP-KLF6 was separated using $4-20 \%$ gradient polyacrylamide gel and then transferred into PVDF membranes. The membrane was blocked with phosphate-buffered saline (PBS) containing 5\% nonfat milk for $1 \mathrm{~h}$ at room temperature and then the membrane was incubated with the indicated primary antibodies at $4{ }^{\circ} \mathrm{C}$ overnight. After washing with PBS containing 0.1\% Tween-20 (PBST), membrane was reacted with HRP-conjugated secondary antibody and the reactive signal was detected using an enhanced chemiluminescence kit (Amersham Pharmacia Biotech, UK). The $\beta$-actin expression was used as the internal control.

\section{Zymography assay}

SAS cells were tranfected with pEGFP or pEGFP-KLF6 and cultured in serum free medium. Twenty-four post-transfection, the conditional medium were collected and subjected into zymography assay. Samples were mixed with loading buffer and were separated by $8 \%$ SDS-polyacrylamide gel containing $0.1 \%$ gelatin. The gel was then washed twice in Zymography washing buffer $(2.5 \%$ Triton $\mathrm{X}-100$ in double distilled $(\mathrm{H} 2 \mathrm{O})$ at room temperature and then incubated at $37{ }^{\circ} \mathrm{C}$ for $12-16 \mathrm{~h}$ in Zymography reaction buffer $(40 \mathrm{mM}$ Tris $-\mathrm{HCl}(\mathrm{pH}$ 8.0), $10 \mathrm{mM} \mathrm{CaCl} 2$, and $0.02 \% \mathrm{NaN} 3$ ). The gel was stained with Coomassie blue R-250 (0.125\% Coomassie blue R-250, 0.1\%amino black, 50\% methanol, and $10 \%$ acetic acid) for $1 \mathrm{~h}$ and destained with methanol/acetic acid/water (20/10/70, v/v/v). 


\section{Migration and invasion assay}

Plasmids such as pLenti-C-mGFP and pLenti-C-mGFP-KLF6 were purchased from Origene. Virus particles were packaged according to the manufacturer's recommendation and infected into SAS cells. Twenty-four hour post-infection, cells were seeded at a density of $5 \times 105 / \mathrm{mL}$ in the upper chamber of the 48-well Boyden chamber. The lower chamber contained 20\% FBS. The chamber was incubated at $37^{\circ} \mathrm{C}$ for $24 \mathrm{~h}$. The cells that migrated to the lower surface of the membrane were fixed in methanol for $10 \mathrm{~min}$ and stained with $10 \%$ Giemsa for $1 \mathrm{~h}$. The migrated cells were pictured at random five fields and counted.

(A)
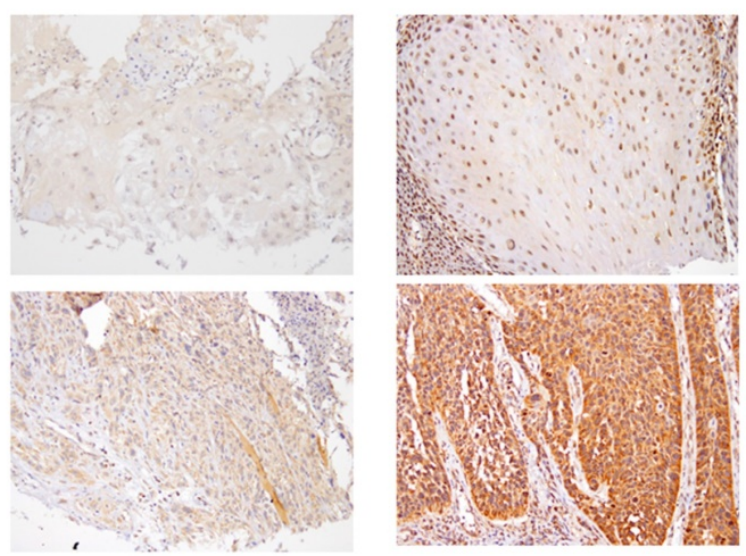

(B)

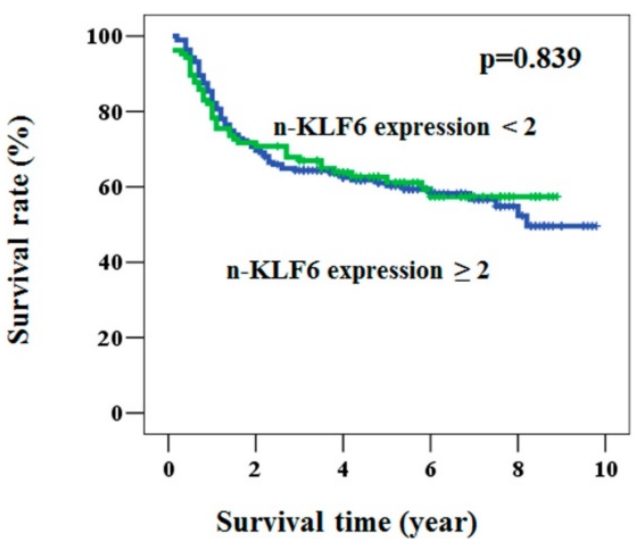

Figure 1. The expression pattern of KLF6 in oral cancer and its relationship with overall survival. (A) The immunohistochemical staining for the expression of KLF6 in oral cancer patients. Upper panel: the expression level < 2. Lower panel: the expression level $\geqq 2$. (B)A Kaplan-Meier survival curve for the oral cancer patients with nuclear expression of KLF6 protein. No significant difference of overall survival rate was found in patients with KLF6 expression < 2 (green line) compared to those with KLF6 expression $\geqq 2$ (blue line) ( $P=$ $0.839)$.

\section{Statistical analysis}

The correlation between the expression levels of KLF6 with different clinicopathological characteristics was measured by a Chi-square test with a Fisher extract test. The overall survival rate was estimated by a Kaplan-Meier plot and log-rank test. Multivariate analysis with a Cox proportional hazard regression model was used to evaluate the prognostic significance of clinical variables. The migration, invasion, and Western blot data are reported as mean $\pm \mathrm{SD}$ of the independent experiments, and were evaluated by Student's t-test. A P value $<0.05$ was considered to be statistically significant.

\section{Results}

\section{The correlation of nuclear KLF6 with clinical parameters in oral cancer patients}

To determine whether KLF6 expression is associated with the clinical parameters of oral cancer patients, we immunohistochemically stained KLF6 through oral cancer tissue microarray. The KLF6 expression levels were subdivided into two categories, namely, low and high, on the basis of the IHC staining intensity, as described in the Materials and Methods section (Figure. 1A). KLF6 was not associated with grade status, 3-year survival, T status, and lymph node metastasis. The nuclear KLF6 expression was significantly lower in oral cancer patients with tumor $>3 \mathrm{~cm}(64.6 \%)$ than in patients with tumor $<3 \mathrm{~cm}(48.5 \%)$. The loss of nuclear KLF6 expression was also correlated with advanced stages (Table 1). Kaplan-Meier survival curve analysis indicated that KLF6 expression was not correlated with the overall survival rate of oral cancer patients (Figure 1B).

Table 1. Relationships of nucleus KLF6 with clinical parameters in oral cancer patients.

\begin{tabular}{|c|c|c|c|c|}
\hline \multirow[t]{2}{*}{ Parameters } & \multirow[t]{2}{*}{ Case number } & \multicolumn{2}{|c|}{ nucleus KLF6 expression } & \multirow[t]{2}{*}{$\mathrm{p}$ value } \\
\hline & & $<2$ & $\geq 2$ & \\
\hline Age (year) & & $55.7 \pm 11.2$ & $55.1 \pm 11.1$ & 0.660 \\
\hline \multicolumn{5}{|l|}{ Gender } \\
\hline Female & 16 & $8(50.0)$ & $8(50.0)$ & \multirow[t]{2}{*}{0.219} \\
\hline Male & 281 & $183(65.1)$ & $98(34.9)$ & \\
\hline \multicolumn{5}{|l|}{ Smoking } \\
\hline No & 77 & $47(60.1)$ & $30(39.0)$ & \multirow[t]{2}{*}{0.482} \\
\hline Yes & 129 & $85(65.9)$ & $44(34.1)$ & \\
\hline \multicolumn{5}{|l|}{ Betal quid } \\
\hline No & 63 & $43(68.3)$ & $20(31.7)$ & \multirow[t]{2}{*}{0.058} \\
\hline Yes & 62 & $32(51.6)$ & $30(48.4)$ & \\
\hline \multicolumn{5}{|c|}{ Differentiation } \\
\hline Well & 44 & $24(54.5)$ & $20(45.5)$ & \multirow[t]{3}{*}{0.216} \\
\hline Moderate & 245 & $163(66.5)$ & $82(33.5)$ & \\
\hline Poor & 8 & $4(50.0)$ & $4(50.0)$ & \\
\hline \multicolumn{5}{|l|}{ Stage } \\
\hline $\mathrm{I}+\mathrm{II}$ & 120 & $66(55.0)$ & $54(45.0)$ & \multirow[t]{2}{*}{0.006} \\
\hline III+IV & 177 & $125(70.6)$ & $52(29.4)$ & \\
\hline \multicolumn{5}{|l|}{$\mathrm{T}$ value } \\
\hline $1+2+3$ & 194 & $121(62.4)$ & $73(37.6)$ & \multirow[t]{2}{*}{0.339} \\
\hline 4 & 103 & $70(68.0)$ & $33(32.0)$ & \\
\hline \multicolumn{5}{|l|}{$\mathrm{N}$ value } \\
\hline 0 & 192 & 118 (61.5) & 74 (38.5) & \multirow[t]{2}{*}{0.165} \\
\hline $1+2+3$ & 105 & 73 (69.5) & $32(30.5)$ & \\
\hline
\end{tabular}

${ }^{1}$ missing 91 cases; ${ }^{2}$ missing 172 cases 
(A)
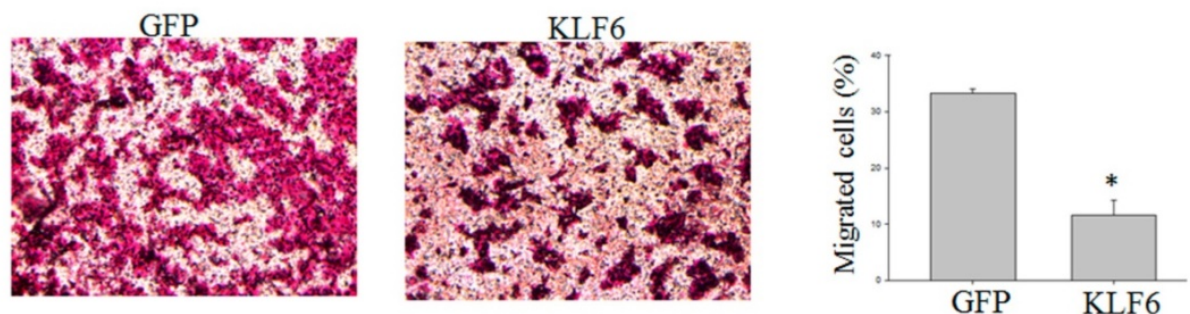

(B)
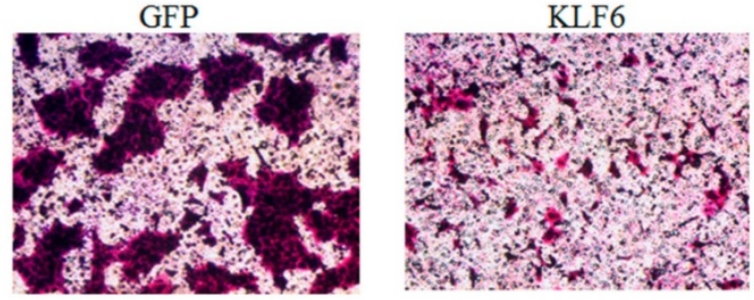

(C)

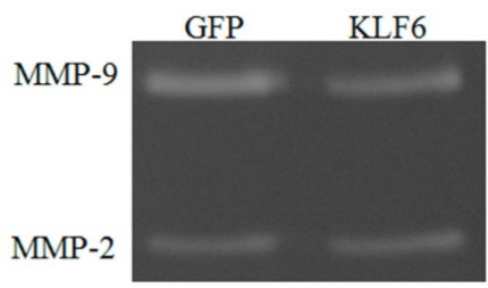

Figure 2. KLF6 attenuated the (A) migration and (B) invasion of oral cancer cells and (C) suppressed MMP-9 activities. SAS cells were infected with pEGFP or PEGFP-KLF6 and subjected to migration (A) or invasion (B) assay. Conditional media derived from SAS cells transfected with pEGFP or pEGFP-KLF6 were subjected to zymography assay. MMP-2 and MMP-9 were indicated. Data represent mean \pm S.D. * and ** denote $\mathrm{P}<0.05$ compared with cells expressing GFP alone.

\section{Overexpression of KLF6 attenuated the migration and inhibited MMP-9 activities of oral cancer cells.}

Previous reports demonstrated that KLF6 is involved in the migration of hepatocellular carcinoma cells [13]. To investigate whether KLF6 also regulates the migration of oral cancer cells, we performed Transwell migration and invasion assay. The migration of SAS cells was significantly reduced by KLF6 overexpression to $15 \%$ lower than that of the cells overexpressing green fluorescent protein (GFP) alone (Figure 2A and B). The invasion of SAS was also attenuated by KLF6 to $40 \%$ lower than that of the cells overexpressing GFP alone.

Matrix metalloproteinases (MMPs) play a critical role in cancer migration and invasion [14]. To determine whether the decreased MMP-9 expression also reduces MMP-9 activity, we conducted a zymography assay and found that KLF6 overexpression significantly decreased the MMP-9 activities. However, evident changes in MMP-2 activities were not found (Figure 2C).

\section{KLF6 downregulated the mesenchymal markers in oral cancer cells}

The dysregulation of EMT is considered a hallmark of cancer metastasis [15]. To evaluate the effects of KLF6 on the expression of EMT markers, we performed Western blot analysis and observed that KLF6 decreased the expression levels of vimentin, snail, and slug to approximately $80 \%, 65 \%$, and $70 \%$, respectively, compared with cells overexpressing GFP alone. However, evident changes in E-cadherin were not found in SAS cells overexpressing GFP or KLF6 (Figure 3).

\section{Discussion}

KLF6 expression is downregulated in several human cancers, such as hepatocellular carcinoma [8], head and neck cancers [7], and gastric cancers [16]. In this study, low nuclear KLF6 expression was correlated with tumor size and advanced stages in oral cancer patients. KLF6 overexpression attenuated the migration and invasion of oral cancer SAS cells. KLF6 also decreased the mRNA level and activities of MMP-9, as evidenced by real-time PCR and zymography, respectively. KLF6 also reduced the mesenchymal marker expression in SAS cells. Therefore, KLF6 may function as a tumor suppressor in oral cancers. In gastric cancer, KLF6 expression is correlated with grade, tumor-node-metastasis stages, and distal metastasis [17]. Low KLF6 expression is also associated with metastasis and 3-year survival rate [17]. In this study, nuclear KLF6 expression was downregulated in advanced stages. However, KLF6 expression was not correlated with the overall survival of oral cancer patients. 


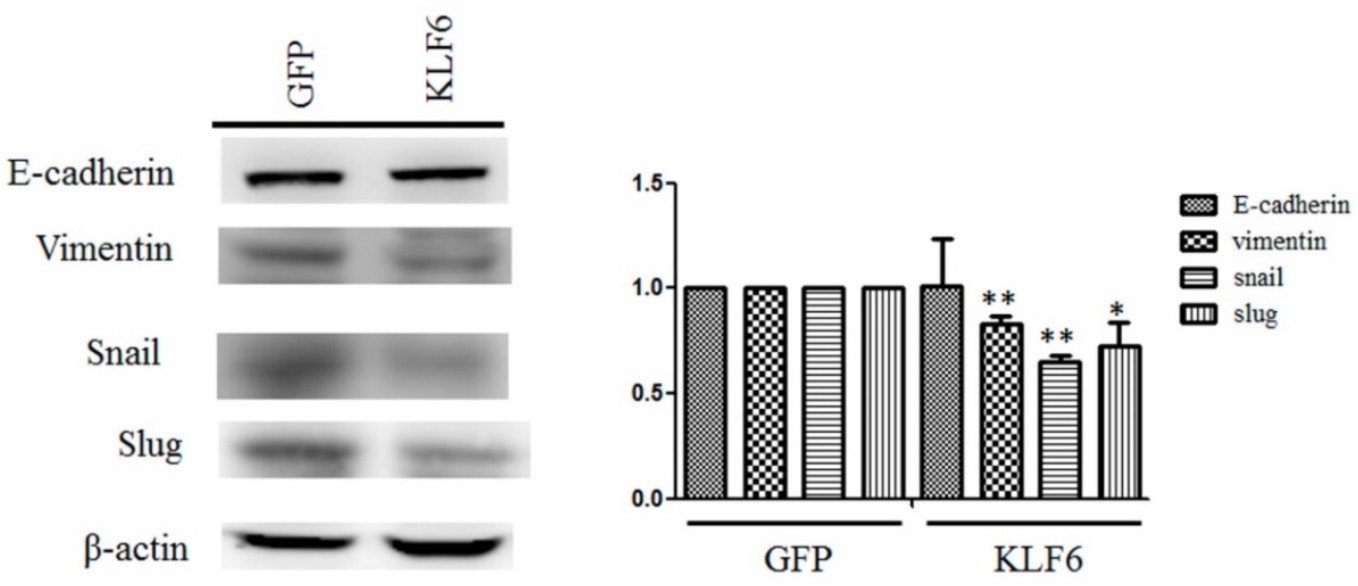

Figure 3. KLF6 reversed the expression of mesenchymal markers. Cell lysates from SAS cell overexpressing PEGFP or pEFGP-KLF6 were examined through Western blot. Right panel: data representing mean \pm S.D. * and ** denote $\mathrm{P}<0.05$ and $\mathrm{P}<0.001$ compared with cells expressing GFP alone, respectively.

In this study, our observation indicated that KLF6 overexpression attenuated the migration and invasion of SAS cells. Zhang et al. revealed that microRNA-181-induced silencing of KLF6 expression promotes the proliferation, migration, and invasion of human gastric cancer SGC-7901 cells [18]. KLF6 overexpression in hepatocellular cancer cell lines decreases cell proliferation and migration [19]. MicroRNA-1301-stimulated KLF6 downregulation also triggers migration and angiogenesis through the reduction of KLF6 expression [20]. Ahronian et al. showed that KLF6 suppresses the expression of VAV3, a Rac1 activator, and subsequently inhibits the metastasis of hepatocellular carcinomas [13]. Micro-RNA-101 also prevents the expression of KLF6 and the proliferation, migration, and invasion in glioblastoma stem cells [21]. Therefore, KLF6 may function as a tumor suppressor gene in oral cancer to prevent migration.

MMPs can degrade extracellular matrixes and trigger cancer cell migration and invasion [22]. Increased MMP expression is also correlated with cancer metastasis [22]. Das et al. performed promoter assay, gel shift, and chromosome immunoprecipitation and found that KLF6 and SP2 bind to the promoter region of MMP-9 and suppress MMP-9 expression in endothelial cells [23]. Furthermore, forced KLF6 expression reduces MMP-9 expression and inhibits osteosarcoma cell invasion [10]. Oncogenic KLF6-SV1 generated from alternative splicing is knocked down and thus decreases MMP-9 expression and as a consequence, the invasion of gastric cancer cells is reduced [24]. Consistent with these observations, zymography assay and real-time PCR analysis respectively revealed that KLF6 overexpression inhibited the activities of MMP-9. Therefore, KLF6 attenuated MMP-9 activities in oral cancer cells.
EMT is a multi-process event that regulates various cellular functions, such as embryogenesis, cell morphology, and cell migration [25]. During EMT, cells remarkably lose epithelial markers, including E-cadherin, and gain mesenchymal markers, such as snail, slug, and vimentin, which subsequently trigger cancer cell migration and invasion [25]. Several KLFs are involved in EMT in cancers. KLF4 overexpression suppresses the snail expression, an EMT protein, in mouse model of breast cancer cells [26]. KLF17 enhances Twist expression and consequently triggers EMT in endometrial cancer [27]. KLF8 promotes the migration and invasion of gastric cancer cells by triggering EMT under hypoxic conditions [28]. In our study, KLF6 inhibited the expression of mesenchymal markers, such as snail, slug, and vimentin. Conversely, Holian et al. demonstrated that KLF6 overexpression stimulates transforming growth factor-induced EMT in human proximal tubule cells, whereas KLF6 knockdown reverses this phenomenon [29]. This difference may be attributed to various cell lines. Therefore, KLF6 could reverse the EMT in oral cancer cells.

In summary, this study revealed the molecular mechanisms of KLF6 in oral cancer cells. Ectopic KLF6 expression decreased the migration and invasion of oral cancer cells. KLF6 also suppressed the expression and activities of MMP-9 and reversed EMT by attenuating the expression of mesenchymal markers. Therefore, our study provided the first evidence that KLF6 functions as a tumor suppressor gene in oral cancer.

\section{Acknowledgments}

This work is supported by NSC 102-2320-B-371-001 from Ministry of Science and Technology of Taiwan. 


\section{Competing Interests}

The authors have declared that no competing interest exists.

\section{References}

1. Black AR, Black JD, Azizkhan-Clifford J. Sp1 and kruppel-like factor family of transcription factors in cell growth regulation and cancer. J Cell Physiol. 2001; 188: 143-60.

2. Kaczynski J, Cook T, Urrutia R. Sp1- and Kruppel-like transcription factors. Genome Biol. 2003; 4: 206.

3. Narla G, Heath KE, Reeves HL, Li D, Giono LE, Kimmelman AC, Glucksman MJ, et al. KLF6, a candidate tumor suppressor gene mutated in prostate cancer. Science. 2001; 294: 2563-6.

4. Chen K, Chen Y, Zhu XD, Bai YS, Wei XZ, Wang CF, Chen ZQ, et al. Expression and significance of Kruppel-like factor 6 gene in osteosarcoma. Int Orthop. 2012; 36: 2107-11.

5. Chiam K, Ryan NK, Ricciardelli C, Day TK, Buchanan G, Ochnik AM, Murti K, et al. Characterization of the prostate cancer susceptibility gene KLF6 in human and mouse prostate cancers. Prostate. 2013; 73: 182-93.

6. Ito G, Uchiyama M, Kondo M, Mori S, Usami N, Maeda O, Kawabe T, et al. Kruppel-like factor 6 is frequently down-regulated and induces apoptosis in non-small cell lung cancer cells. Cancer Res. 2004; 64: 3838-43.

7. Teixeira MS, Camacho-Vanegas O, Fernandez Y, Narla G, DiFeo A, Lee B, Kalir T, et al. KLF6 allelic loss is associated with tumor recurrence and markedly decreased survival in head and neck squamous cell carcinoma. Int J Cancer. 2007; 121: 1976-83.

8. Song J, Kim CJ, Cho YG, Kim SY, Nam SW, Lee SH, Yoo NJ, et al. Genetic and epigenetic alterations of the KLF6 gene in hepatocellular carcinoma. J Gastroenterol Hepatol. 2006; 21: 1286-9.

9. Tarocchi M, Hannivoort R, Hoshida Y, Lee UE, Vetter D, Narla G, Villanueva $\mathrm{A}$, et al. Carcinogen-induced hepatic tumors in KLF6+/- mice recapitulate aggressive human hepatocellular carcinoma associated with p53 pathway deregulation. Hepatology. 2011; 54: 522-31.

10. Jianwei Z, Enzhong B, Fan L, Jian L, Ning A. Effects of Kruppel-like factor 6 on osteosarcoma cell biological behavior. Tumour Biol. 2013; 34: 1097-105.

11. Argiris A, Karamouzis MV, Raben D, Ferris RL. Head and neck cancer. Lancet. 2008; 371: 1695-709.

12. Chen CJ, Hsu LS, Lin SH, Chen MK, Wang HK, Hsu JD, Lee H, et al. Loss of nuclear expression of Kruppel-like factor 4 is associated with poor prognosis in patients with oral cancer. Hum Pathol. 2012; 43: 1119-25.

13. Ahronian LG, Zhu LJ, Chen YW, Chu HC, Klimstra DS, Lewis BC. A novel KLF6-Rho GTPase axis regulates hepatocellular carcinoma cell migration and dissemination. Oncogene. 2016; 35: 4653-62.

14. Egeblad M, Werb Z. New functions for the matrix metalloproteinases in cancer progression. Nat Rev Cancer. 2002; 2: 161-74

15. $\mathrm{Li} \mathrm{L}, \mathrm{Li} \mathrm{W}$. Epithelial-mesenchymal transition in human cancer: comprehensive reprogramming of metabolism, epigenetics, and differentiation. Pharmacol Ther. 2015; 150: 33-46.

16. Wei D, Gong W, Kanai M, Schlunk C, Wang L, Yao JC, Wu TT, et al. Drastic down-regulation of Kruppel-like factor 4 expression is critical in human gastric cancer development and progression. Cancer Res. 2005; 65: 2746-54.

17. Zhang Q, Tan XP, Yuan YS, Hu CM, He CH, Wang WZ, Li JC, et al. Decreased expression of KLF6 and its significance in gastric carcinoma. Med Oncol. 2010; 27: 1295-302.

18. Zhang X, Nie Y, Du Y, Cao J, Shen B, Li Y. MicroRNA-181a promotes gastric cancer by negatively regulating tumor suppressor KLF6. Tumour Biol. 2012; 33: 1589-97.

19. Wen PH, Wang DY, Zhang JK, Wang ZH, Pan J, Shi XY, Yang H, et al. Kruppel-like factor 6 suppresses growth and invasion of hepatocellular carcinoma cells in vitro and in vivo. Int J Immunopathol Pharmacol. 2016;

20. Liang WC, Wang Y, Xiao LJ, Wang YB, Fu WM, Wang WM, Jiang HQ, et al. Identification of miRNAs that specifically target tumor suppressive KLF6-FL rather than oncogenic KLF6-SV1 isoform. RNA Biol. 2014; 11: 845-54.

21. Yao YL, Ma J, Wang P, Xue YX, Li Z, Zhao LN, Li ZQ, et al. miR-101 acts as a tumor suppressor by targeting Kruppel-like factor 6 in glioblastoma stem cells. CNS Neurosci Ther. 2015; 21: 40-51.

22. Itoh $Y$, Nagase H. Matrix metalloproteinases in cancer. Essays Biochem. 2002; 38: 21-36.

23. Das A, Fernandez-Zapico ME, Cao S, Yao J, Fiorucci S, Hebbel RP, Urrutia R, et al. Disruption of an SP2/KLF6 repression complex by SHP is required for farnesoid X receptor-induced endothelial cell migration. J Biol Chem. 2006; 281: 39105-13.

24. Chen H, Chen L, Sun L, Zhen H, Li X, Zhang Q. A small interfering RNA targeting the KLF6 splice variant, KLF6-SV1, as gene therapy for gastric cancer. Gastric Cancer. 2011; 14: 339-52.

25. Przybyla L, Muncie JM, Weaver VM. Mechanical Control of Epithelial-to-Mesenchymal Transitions in Development and Cancer. Annu Rev Cell Dev Biol. 2016; 32. 527-554

26. Yori JL, Seachrist DD, Johnson E, Lozada KL, Abdul-Karim FW, Chodosh LA, Schiemann WP, et al. Kruppel-like factor 4 inhibits tumorigenic progression and metastasis in a mouse model of breast cancer. Neoplasia. 2011; 13: 601-10.
27. Dong $\mathrm{P}$, Kaneuchi $\mathrm{M}$, Xiong $\mathrm{Y}$, Cao L, Cai M, Liu X, Guo SW, et al. Identification of KLF17 as a novel epithelial to mesenchymal transition inducer via direct activation of TWIST1 in endometrioid endometrial cancer. Carcinogenesis. 2014; 35: 760-8

28. Liu N, Wang Y, Zhou Y, Pang H, Zhou J, Qian P, Liu L, et al. Kruppel-like factor 8 involved in hypoxia promotes the invasion and metastasis of gastric cancer via epithelial to mesenchymal transition. Oncol Rep. 2014; 32: 2397-404.

29. Holian J, Qi W, Kelly DJ, Zhang Y, Mreich E, Pollock CA, Chen XM. Role of Kruppel-like factor 6 in transforming growth factor-beta1-induced epithelial-mesenchymal transition of proximal tubule cells. Am J Physiol Renal Physiol. 2008; 295: F1388-96. 